\title{
Frailty, ageing and inflammation: reality and perspectives
}

\author{
Eugenio Mocchegiani · Andrea Corsonello • \\ Fabrizia Lattanzio
}

Received: 6 August 2010/Accepted: 10 August 2010/Published online: 18 August 2010

(C) Springer Science+Business Media B.V. 2010

The knowledge about frailty is rapidly growing, and improved understanding of molecular and physiological mechanisms involved in determining the increased vulnerability of frail older adults has encouraged investigators from many disciplines to contribute to this emerging field of research.

Medical practitioners have often used the term "frailty" to characterize the weakest and most vulnerable subset of older adults. Recent research efforts have helped to better define clinical and physiological characteristics of frailty and to highlight the vulnerability of frail older adults to poor health outcomes. The term "frail" has various clinically relevant meanings (e.g., likely to fail or die quickly; unusually susceptible to some diseases or other infirmity; lacking normal strength or force; weak, thin, and slight). Although there is a wide range of common phenotypes in geriatric medicine to which such terms

E. Mocchegiani $(\square)$

Ctr. Nutrition and Ageing, Italian National Research Centre on Aging (INRCA), Via Birarelli 8, 60121 Ancona, Italy

e-mail: e.mocchegiani@inrca.it

\section{A. Corsonello}

Unit of Geriatric Pharmacoepidemiology, Italian National

Research Centre on Aging (INRCA), Cosenza, Italy

F. Lattanzio

Scientific Direction, Italian National Research

Centre on Aging (INRCA), Ancona, Italy may be related, including muscle weakness, bone fragility, very low body mass index, susceptibility to falling, vulnerability to trauma, vulnerability to infection, high risk for delirium, blood pressure instability, and severely diminished physical capabilities, the word "frailty" in the common notation of clinical geriatric medicine usually describes a condition in which a critical combination of these specific impairments occurs. Nevertheless, researchers have applied different approaches describing these phenomena. As reported by Rockwood (2005), conceptual definitions of frailty have included a variety of domains with a multidisciplinary approach, including physical performance, cognitive function, psychological factors, as well as disability owing to the presence of illness. Other investigators defined frailty as a single syndrome coupled with the presence of clinical traits associated with aging, such as weakness, fatigue, weight loss, decreased balance, low levels of physical activity, slowed motor processing and performance, social withdrawal, mild cognitive changes, and increased vulnerability to stressors (Fried et al. 2009). Whatever is the definition of frailty we could consider, there is a general consensus that frailty is also characterized by specific alterations in physiological variables, including inflammatory biomarkers, altered glucose metabolism, mitochondria function and markers of clotting processes, providing further evidence of multisystemic involvement (Fried et al. 2009).

Therefore, the old frail subject has to be considered as the sum of various patho-physiological components 

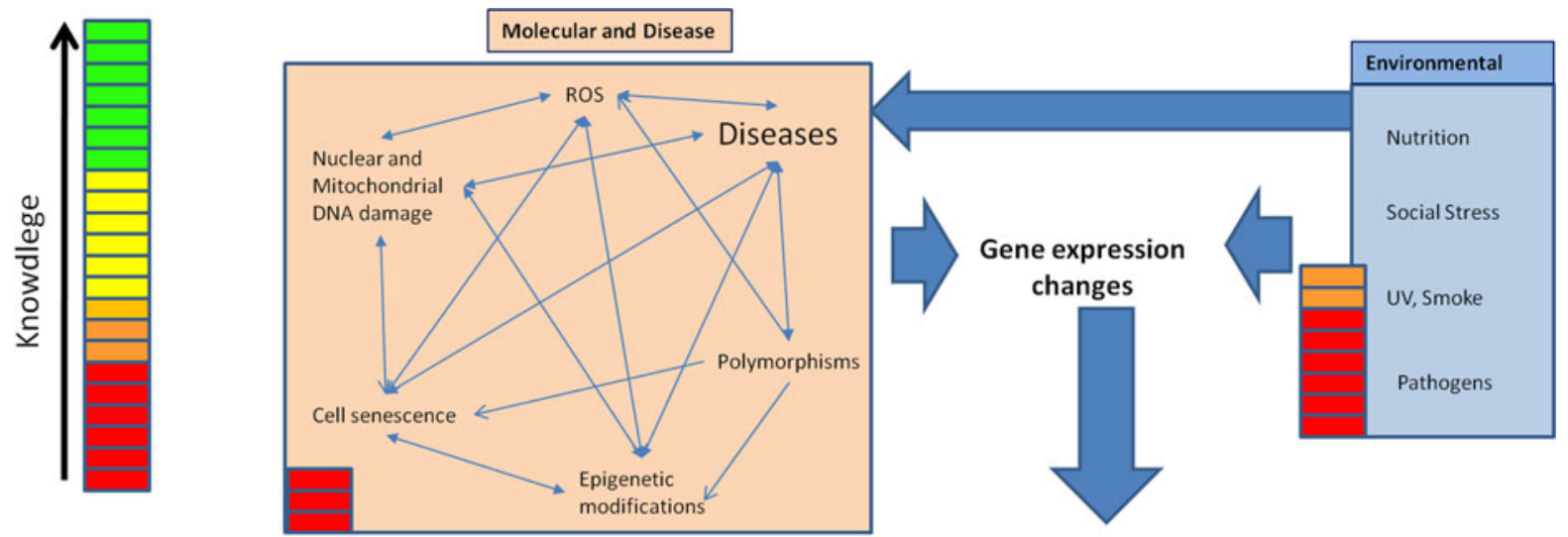
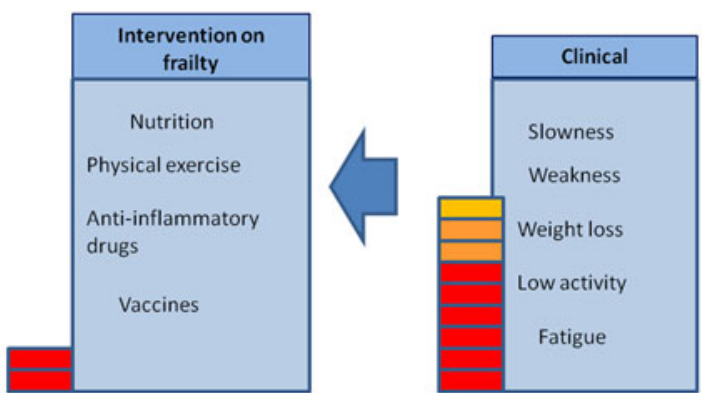

Fig. 1 Degree of knowledge of the relationship between molecular, patho-physiology, and clinical variables that may constitute frailty in old people and specific interventions discussed in the present issue. Both intrinsic (molecular) and environmental factors may lead to the appearance of disability owing to age-related diseases. These factors directly or indirectly affect the gene expression of genes mainly related to the inflammation and neuroendocrine pathway with

leading to a status of vulnerability and predisposing to the appearance of disability and degenerative diseases, such as sarcopenia, cardiovascular diseases, dementia, and cancer. In this special issue, we aimed at summarizing the state of the art on many of these aspects. We considered low grade chronic inflammation, as well as reduced capacity to mount an efficient stress-related response, mitochondria dysfunction, insulin resistance, altered endocrine pathways and central nervous system failure the main background for frailty. We also aimed at reviewing the available evidence about specific potential treatment and prevention options against frailty. By bringing together clinical and basic science investigators from a wide variety of aging research fields, this special issue represents a very interesting multidisciplinary attempt to summarize the state of the art in frailty research. One of the key

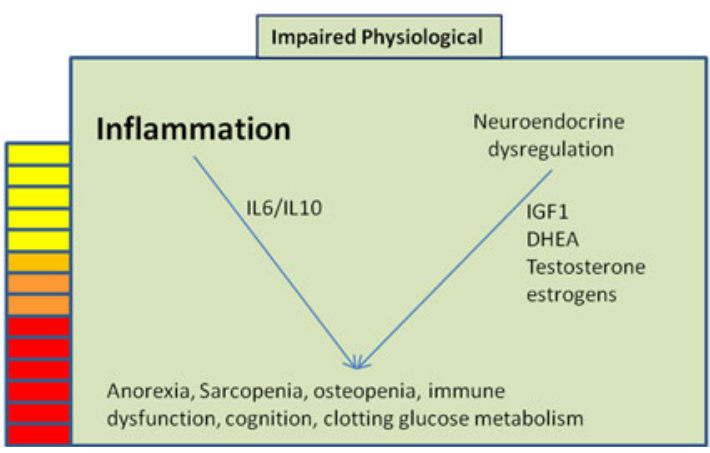

subsequent impaired physiological functions and the arising of some diseases related to frailty (anorexia, sarcopenia, osteopenia, immunodeficiency) that lead to series of clinical manifestations (weakness, slowness, weight loss, low activity, fatigue) characteristic of the frailty syndrome and the subsequent possible interventions. Explanation: Red, low knowledge; Orange-Yellow, medium knowledge; Green, good knowledge

messages from this special issue is that many of the physiological systems thought to decline in frailty function is communication. Indeed, the evidence presented here strongly suggests that the interaction between inflammatory pathways, bioactive hormones, mitochondria, nutrition and genes are of critical importance in molecular and physiological changes that may contribute to the development of frailty and age-related chronic diseases. Such a conceptual framework is of particular interest because it may help investigators to identify crucial triggering mechanisms that lead to multisystem decline in frailty. Figure 1 summarizes the actual degree of knowledge about molecular, environmental, physiological and clinical changes that may contribute to frailty as reported in this special issue and the future directions for putative interventions to prevent frailty in old people. 


\section{References}

Fried LP, Xue QL, Cappola AR, Ferrucci L, Chaves P, Varadhan R, Guralnik JM, Leng SX, Semba RD, Walston JD, Blaum CS, Bandeen-Roche K (2009) Nonlinear multisystem physiological dysregulation associated with frailty in older women: implications for etiology and treatment. J Gerontol A Biol Sci Med Sci 64:1049-1057 Rockwood K (2005) Frailty and its definition: a worthy challenge. J Am Geriatr Soc 53:1069-1070 